\section{Distribuição espacial do baixo peso ao nascer em Sergipe, Brasil, 1995/1998}

\author{
Space distribution of low birth weight \\ in Sergipe, Brazil, 1995/1998
}

\author{
1 Núcleo de Pós-graduação \\ em Medicina, Universidade \\ Federal de Sergipe, \\ Aracaju, Brasil. \\ 2 Núcleo de Pós-graduação \\ em Geografia, Universidade \\ Federal de Sergipe, \\ Aracaju, Brasil. \\ 3 Departamento de \\ Estatística, Universidade \\ Federal de Sergipe, \\ Aracaju, Brasil. \\ Correspondência \\ R. Q. Gurgel \\ Núcleo de Pós-graduação \\ em Medicina, Universidade \\ Federal de Sergipe. \\ Av. Beira Mar 2016, \\ apto. 402, Aracaju, SE \\ 49025-040, Brasil. \\ ricardoqg@infonet.com.br
}

\begin{abstract}
This study analyzes the spatial distribution of low birth weight $(L B W)$ in the State of Sergipe, Brazil, evaluating the event as a health indicator. The analysis was based on 166,312 live births in the 75 municipalities in the State, using an ecological approach. Four methods were used: cluster analysis, correlation analysis, multiple regression analysis, and multiple comparisons (Tukey). The study shows internal homogeneity in the municipalities' health districts and in the groups resulting from the cluster analysis. Considering a 10\% loss of information, seven groups were identified, two of which were residues (Aracaju and Porto da Folha). There is a downward trend in LBW in Sergipe (with a mean of $6.97 \%$ during the period), reflecting $a$ slight improvement in quality of life. The causes suggest relations with the environment and are influenced by spatial occupation patterns. The percentage of $L B W$ is a useful indicator of individual risk, but as a collective health indicator it does not appear capable of expressing differences between regions that do not display strong inter-variability.
\end{abstract}

Spatial Distribution; Low Birth Weight; Equity
Ricardo Queiroz Gurgel 1 Iane Martha Oliveira Dias 1 Vera Lúcia Alves França ${ }^{2}$ Daniel Francisco Neyra Castañeda ${ }^{3}$

\section{Introdução}

O baixo peso ao nascer tem sido reconhecido como a condição de sobrevivência mais importante quando relacionada com a morbi-mortalidade no primeiro ano de vida 1,2,3,4,5.

O estudo do baixo peso ao nascer pode mostrar evidências da atuação de fatores de natureza diversa sobre o potencial genético individual, sendo sua distribuição diferente e específica para populações distintas, em função, principalmente, das condições de vida, podendo ser um bom indicador de saúde 6 .

Os índices do baixo peso ao nascer no mundo variam de 3,00 a 43,00\% 7. Essa ampla variação tem sua explicação muito mais nas disparidades sócio-econômicas que em possíveis diferenças genéticas 8 .

$\mathrm{O}$ baixo peso ao nascer é resultante de influências biológicas, sócio-econômicas e nutricionais que acontecem antes da gravidez, continuam durante a gestação e parto, e ao entrar em ação recíproca com os fatores obstétricos podem complicar o produto final da gestação. Não obstante a multiplicidade de fatores de risco do baixo peso ao nascer, cada vez mais há possibilidades de se constatar, antes do nascimento, quais crianças estarão expostas a um risco elevado no curso da primeira semana e, até, do restante do primeiro ano de vida. A relação entre o déficit ponderal ao nascer e as condições sócio-econômicas da 
comunidade está, cada vez mais, sendo estabelecida 6 .

O estudo do baixo peso ao nascer como indicador de saúde, das condições de vida e de seus impactos sobre a situação de saúde da população em geral, e de grupos sociais, em particular, têm merecido atenção crescente no campo da saúde coletiva. Segundo Victora et al. 9, que ao discutirem alternativas no uso do coeficiente de mortalidade infantil, dizem ser necessário buscar um indicador de saúde que ainda reflita com precisão a evolução da qualidade de vida. Tal indicador deve ser sensível a mudanças nas condições básicas da existência (incluindo nutrição, habitação, saneamento) e, também, relativamente influenciado por ações médico-sanitárias específicas.

O baixo peso ao nascer e o ambiente são intrinsecamente influenciados pelos padrões de ocupação do espaço, pela forma como os indivíduos se inserem nos substratos de renda da população, sendo necessário localizar onde se concentram situações sociais mais vulneráveis. Sendo assim, o estudo do contexto sócioeconômico é beneficiado pela incorporação da distribuição espacial dos eventos de saúde, incluindo o baixo peso ao nascer.

A medida do crescimento intra-uterino e do baixo peso ao nascer, para a medicina em nível individual, está bem estabelecida 5,10 , no entanto, este estudo se propõe a avaliar a sua importância como indicador coletivo de saúde e de qualidade de vida.

O presente artigo faz uso de indicadores tradicionalmente utilizados por várias instituições como o Banco Mundial e a Organização das Nações Unidas, comparando-os ao índice de baixo peso ao nascer.

Este estudo tem como objetivo analisar a distribuição espacial do baixo peso ao nascer em Sergipe, Brasil (1995/1998), avaliando o evento como indicador de saúde e de qualidade de vida, além de conhecer o perfil desse indicador nos municípios sergipanos.

\section{Material e métodos}

O estudo focaliza a população e o evento por meio de uma abordagem ecológica, apresentando uma retrospectiva de uma série de quatro anos de nascimentos vivos com baixo peso $(<2.500 \mathrm{~g})$. A análise foi feita a partir de 166.312 nascimentos vivos ocorridos no período de 1995/1998, nos 75 municípios do Estado de Sergipe. Para o cálculo foram utilizados dados do Sistema de Informação de Nascidos Vivos (SINASC), sendo agrupados por município de residência da mãe, por distritos de saúde para Sergipe, ano a ano e no conjunto dos quatro anos (1995/1998).

A análise por Distritos Regionais de Saúde (DRS) obedeceu ao agrupamento de municípios determinado pelo Modelo Assistencial de Sergipe 11. Os sete grupos de municípios tinham como sedes regionais: Aracaju, Itabaiana, Capela, Propriá, Nossa Senhora da Glória, Lagarto e Estância.

O Estado de Sergipe ocupa uma área de $22.050 \mathrm{~km}^{2}$, o que corresponde a $2,80 \%$ do território da Região Nordeste, e conta com uma população de 1.784.475 habitantes 12, correspondente a 3,63\% da população nordestina e 1,03\% da população do Brasil.

Para análise foi desenvolvida uma matriz de correlação utilizando indicadores disponíveis como: percentual de baixo peso ao nascer, Índice de Desenvolvimento Humano (IDH), Índice de Desenvolvimento Infantil (IDI), Coeficiente de Mortalidade Infantil (CMI), percentual de cobertura pré-natal (gestantes com no mínimo seis consultas), percentual de vacinação infantil, de moradores com abastecimento de água tratada e percentual de alfabetização. Nessa análise de distribuição espacial foram utilizados quatro métodos estatísticos: análise de agrupamento, análise de correlação, análise de regressão múltipla e comparações múltiplas Tukey-HSD ${ }^{13}$. A análise foi realizada para o conjunto dos 75 municípios sergipanos, usando-se a análise de agrupamento (clusters analysis). Assim, foi observada a capacidade discriminatória dos indicadores usados, no sentido de separar os municípios em grupos cujas características pudessem refletir a homogeneidade interna de seus componentes ou a heterogeneidade entre membros dos diferentes grupos de municípios, por distritos de saúde e grupos resultantes do dendrograma. Como resultante da análise de agrupamento, o dendrograma apresentou os grupos de municípios similares, considerando níveis de perda de informação em torno de $10,00 \%$.

Essa análise foi realizada calculando-se a distância euclidiana média entre os municípios, com o objetivo de separá-los em conglomerados ou clusters com o uso do algoritmo UPGMA (Unweighted Pair Group Method with Arithmetic Mean). O método análise de correlação proporcionou observar o grau de relação entre as variáveis. Para estabelecer uma comparação entre os distritos de saúde e os grupos resultantes do dendrograma foi utilizada a análise de regressão múltipla e de comparações múltiplas. O método de comparação de média (multiple comparisons) é uma técnica incorpo- 
rada dentro da análise de variância (ANOVA). O nível de significância adotado foi de 5\%. Como as variáveis não apresentam a mesma escala e/ou unidade, foi utilizada uma transformação estatística de modo a torná-las adimensionais e com variação de intervalo de 0 a 1 . Os dados de cada variável foram transformados em uma distribuição normal padrão em que a média é igual a 0 e a variância a 1 . Uma vez padronizadas, procederam-se as comparações.

O mapeamento da distribuição espacial do baixo peso ao nascer foi feito utilizando o geoprocessamento, por meio do Sistema de Informações Geográficas, ATLAS GIS. Como resultado final dessa análise, os 75 municípios foram representados utilizando-se as variáveis resultantes do processo, o que facilitou a análise espacial do evento.

\section{Resultados}

Em Sergipe, nos últimos dez anos, o percentual do baixo peso ao nascer vem apresentando taxas declinantes. No período estudado (1995/ 1998) constatou-se que a média do evento foi de $6,97 \%$. Em 1995, 7,84\% e no final do período analisado 7,04\%, havendo uma redução de $34,80 \%$ (Tabela 1).

Entre os DRS de Sergipe não houve diferença estatisticamente significativa para o baixo peso ao nascer, apesar da região metropolitana de Aracaju (DRS7) apresentar o maior índice, $7,65 \%$. Contraditoriamente, essa região também apresenta os maiores IDH $(0,521)$ e taxa de alfabetização (80,47\%), o terceiro maior IDI $(0,499)$ e a segunda menor taxa de mortalidade infantil $(59,77 \%)$. Os DRS 7,2 e 1 que tinham os maiores percentuais de baixo peso ao nascer tiveram também os maiores IDH e menores taxas de mortalidade infantil. A média geral de realização de pré-natal foi baixa, sendo que os DRS 6 e 1 apresentaram percentuais maiores que os demais. A taxa de vacinação foi semelhante entre todos os DRS, dentro de percentuais considerados aceitáveis (Tabela 2).

\begin{tabular}{ll} 
Tabela 1 & \\
\hline & \\
Evolução do baixo peso ao nascer. \\
Sergipe, Brasil, 1988/1998. \\
\hline \multicolumn{2}{c}{ Baixo peso ao nascer (\%) } \\
\hline Ano & 10,80 \\
1988 & 10,60 \\
1989 & 9,10 \\
1994 & 7,84 \\
1995 & 6,89 \\
1996 & 6,91 \\
1998 & 7,04
\end{tabular}

Fonte: Ministério da Saúde 14.

Valores médios das variáveis originais inferidos na análise de agrupamentos e dos componentes principais por Distritos Regionais de Saúde de Sergipe, Brasil. Diferença estatística entre os grupos para cada variável. Modelo de comparações múltiplas (Tukey-HSD), intervalo de confiança de $95 \%$.

\begin{tabular}{|c|c|c|c|c|c|c|c|c|}
\hline \multirow[t]{2}{*}{ Distritos } & \multicolumn{7}{|c|}{ Regionais de Saúde (DRS) } & \multirow[t]{2}{*}{ Comparação } \\
\hline & 7 & 2 & 1 & 6 & 4 & 3 & 5 & \\
\hline Baixo peso ao nascer (\%) & 7,65 & 7,04 & 6,59 & 6,54 & 6,42 & 6,35 & 6,17 & DRS7 $=$ DRS2 $=$ DRS1 $=$ DRS6 $=$ DRS4 $=$ DRS3 = DRS5 \\
\hline $\begin{array}{l}\text { Índice de } \\
\text { desenvolvimento } \\
\text { humano }\end{array}$ & 5,21 & 4,14 & 4,10 & 3,85 & 3,64 & 4,40 & 3,84 & $(\mathrm{DRS7}=\mathrm{DRS} 2=\mathrm{DRS} 1)>(\mathrm{DRS} 6=\mathrm{DRS} 4=\mathrm{DRS} 3=\mathrm{DRS} 5)$ \\
\hline $\begin{array}{l}\text { Índice de } \\
\text { desenvolvimento } \\
\text { infantil }\end{array}$ & 4,99 & 4,71 & 4,81 & 4,76 & 4,27 & 5,39 & 5,22 & $(\mathrm{DRS} 3=\mathrm{DRS} 1=\mathrm{DRS} 2=\mathrm{DRS} 5=\mathrm{DRS} 6=\mathrm{DRS} 7)>(\mathrm{DRS} 4)$ \\
\hline $\begin{array}{l}\text { Taxa de mortalidade } \\
\text { infantil }\end{array}$ & 59,77 & 57,29 & 62,01 & 65,71 & 76,53 & 76,33 & 74,82 & $(\mathrm{DRS} 4=\mathrm{DRS} 3=\mathrm{DRS} 5=\mathrm{DRS} 6)>(\mathrm{DRS} 1=\mathrm{DRS} 7=\mathrm{DRS} 2)$ \\
\hline Pré-natal (\%) & 25,49 & 16,44 & 34,39 & 44,38 & 18,91 & 21,69 & 16,63 & $(\mathrm{DRS6}=\mathrm{DRS} 1)>(\mathrm{DRS7}=\mathrm{DRS} 3=\mathrm{DRS} 4=\mathrm{DRS} 5=\mathrm{DRS} 2)$ \\
\hline Vacinação infantil (\%) & 88,48 & 93,69 & 96,11 & 99,51 & 84,61 & 90,64 & 94,49 & DRS6 $=$ DRS1 $=$ DRS2 $=$ DRS5 $=$ DRS3 $=$ DRS7 $=$ DRS4 \\
\hline Moradores com água (\%) & 5,89 & 87,88 & 87,30 & 87,08 & 85,14 & 86,48 & 88,67 & DRS5 $=$ DRS2 $=$ DRS1 $=$ DRS6 $=$ DRS3 $=$ DRS4 $=$ DRS7 \\
\hline Taxa de alfabetização & 80,47 & 65,65 & 66,46 & 66,24 & 64,15 & 74,02 & 69,24 & $($ DRS7 $=$ DRS3 $)>($ DRS5 $=$ DRS1 $=$ DRS6 $=$ DRS2 $=$ DRS4 $)$ \\
\hline
\end{tabular}

Fonte: Instituto Brasileiro de Geografia e Estatística 12; Ministério da Saúde 14; Fundo das Nações Unidas para a Infância 23;

Empresa de Desenvolvimento Sustentável do Estado de Sergipe, Secretaria de Planejamento e Tecnologia de Sergipe 24. 
A análise de agrupamento teve como resultado a formação de sete grupos, sendo que dois eram resíduos formados, respectivamente, por Aracaju e Porto da Folha. Assim, os cinco grupos restantes apresentaram composição diversa. O Grupo 1 é formado por 19 municípios de diferentes portes populacionais distribuídos por diversas áreas do Estado (Simão Dias, Tobias Barreto, Santa Luzia do Itanhy, Poço Verde, Riachão do Dantas, Pacatuba, Umbaúba, Siriri, Rosário do Catete, Ribeirópolis, São Francisco, Santa Rosa de Lima, Santo Amaro das Brotas, Riachuelo, Pirambu, Pinhão, Pedra Mole, São Cristóvão e Nossa Senhora do Socorro). O Grupo 2 ficou constituído por 12 municípios (Telha, Tomar do Geru, São Miguel do Aleixo, São Domingos, Salgado, Propriá, Pedrinhas, Nossa Senhora das Dores, Nossa Senhora de Lourdes, Muribeca, Neópolis e Malhada dos Bois). O Grupo 3 conta com nove municípios (Poço Redondo, Nossa Senhora Aparecida, Monte Alegre de Sergipe, Japoatã, Itaporanga d'Ajuda, Indiaroba, Gracho Cardoso, Gararu e Carira). O Grupo 4 ficou constituído por 22 municípios, com destaque para alguns subcentros regionais como Estância, Lagarto, Itabaiana e Nossa Senhora da Glória, além de Maruim, Laranjeiras, Japaratuba, Moita Bonita, Malhador, Macambira, Itabi, Feira Nova, Carmópolis, General Maynard, Divina Pastora, Frei Paulo, Capela, Itabaianinha, Boquim, Campo do Brito, Areia Branca e
Poço Verde. Por fim, o Grupo 5 ficou formado por 12 municípios: Santana de São Francisco, Canindé de São Francisco, Barra dos Coqueiros, Cedro de São João, Ilha das Flores, Cumbe, Cristinápolis, Canhoba, Brejo Grande, Arauá, Aquidabã e Amparo do São Francisco (Tabela 3 e Figura 1).

Não houve diferença estatisticamente significativa entre esses grupos em relação ao baixo peso ao nascer, sendo que os maiores percentuais foram observados em Aracaju e no Grupo 3 (Tabela 3). Esse grupo também apresentava os menores IDH $(0,345)$ e IDI $(0,405) \mathrm{e}$ a segunda maior taxa de mortalidade infantil (70,79\%). Nas outras variáveis não houve diferenças entre os grupos.

\section{Discussão}

O baixo peso ao nascer, em Sergipe, apresenta tendência declinante, semelhante ao que ocorre no restante do Brasil. No período estudado o percentual médio foi de $6,97 \%$. Dados do SINASC/DATASUS 14 confirmam que o estado apresenta um dos menores índices do Nordeste, assumindo, portanto, posição privilegiada frente aos demais: Bahia $(7,48 \%)$, Alagoas $(7,90 \%)$, Paraíba $(7,42 \%)$ e Piauí $(7,23 \%)$. Sua capital, Aracaju, com 7,39\%, apresenta um dos menores índices de baixo peso ao nascer dentre as capi-

Tabela 3

Valores médios das variáveis originais inferidos na análise de agrupamentos e dos componentes principais por grupos resultantes do dendrograma (clusters). Diferença estatística entre os grupos para cada variável. Modelo de comparações múltiplas (Tukey-HSD), intervalo de confiança de 95\%.

\begin{tabular}{|c|c|c|c|c|c|c|}
\hline & \multicolumn{5}{|c|}{ Grupos por dendrograma (G) } & \multirow[t]{2}{*}{ Comparação } \\
\hline & 3 & 4 & 5 & 2 & 1 & \\
\hline Baixo peso ao nascer (\%) & 7,20 & 7,09 & 6,43 & 6,08 & 6,06 & $\mathrm{G} 3=\mathrm{G} 4=\mathrm{G} 5=\mathrm{G} 2=\mathrm{G} 1$ \\
\hline $\begin{array}{l}\text { Índice de } \\
\text { desenvolvimento } \\
\text { humano }\end{array}$ & 3,45 & 4,46 & 3,92 & 4,03 & 4,14 & $(\mathrm{G} 4=\mathrm{G} 1=\mathrm{G} 2)>(\mathrm{G} 3=\mathrm{G} 5)$ \\
\hline $\begin{array}{l}\text { Índice de } \\
\text { desenvolvimento } \\
\text { infantil }\end{array}$ & 4,05 & 5,17 & 4,57 & 5,01 & 5,14 & $(\mathrm{G} 4=\mathrm{G} 1=\mathrm{G} 2)>(\mathrm{G} 5=\mathrm{G} 3)$ \\
\hline $\begin{array}{l}\text { Taxa de mortalidade } \\
\text { infantil }\end{array}$ & 70,79 & 65,77 & 71,09 & 68,54 & 68,76 & $\mathrm{G} 5=\mathrm{G} 3=\mathrm{G} 1=\mathrm{G} 2=\mathrm{G} 4$ \\
\hline Pré-natal (\%) & 17,89 & 26,83 & 22,47 & 24,28 & 30,38 & $\mathrm{G} 1=\mathrm{G} 4=\mathrm{G} 5=\mathrm{G} 2=\mathrm{G} 3$ \\
\hline Vacinação infantil (\%) & 86,85 & 91,36 & 92,13 & 93,75 & 94,92 & $\mathrm{G} 1=\mathrm{G} 2=\mathrm{G} 5=\mathrm{G} 4=\mathrm{G} 3$ \\
\hline Moradores com água (\%) & 87,17 & 86,51 & 83,70 & 89,73 & 83,49 & $\mathrm{G} 2=\mathrm{G} 3=\mathrm{G} 4=\mathrm{G} 5=\mathrm{G} 1$ \\
\hline Taxa de alfabetização & 62,31 & 71,05 & 68,53 & 68,38 & 69,95 & $(\mathrm{G} 4=\mathrm{G} 1=\mathrm{G} 2=\mathrm{G} 5)>(\mathrm{G} 3)$ \\
\hline
\end{tabular}

Fonte: Instituto Brasileiro de Geografia e Estatística 12; Ministério da Saúde 14; Fundo das Nações Unidas para a Infância 23; Empresa de Desenvolvimento Sustentável do Estado de Sergipe, Secretaria de Planejamento e Tecnologia de Sergipe 24. 
Figura 1

Análise de agrupamento dos 75 municípios do Estado de Sergipe, Brasil. Modelo cluster com distância euclidiana Método Ward's, dados padronizados em todas as variáveis.

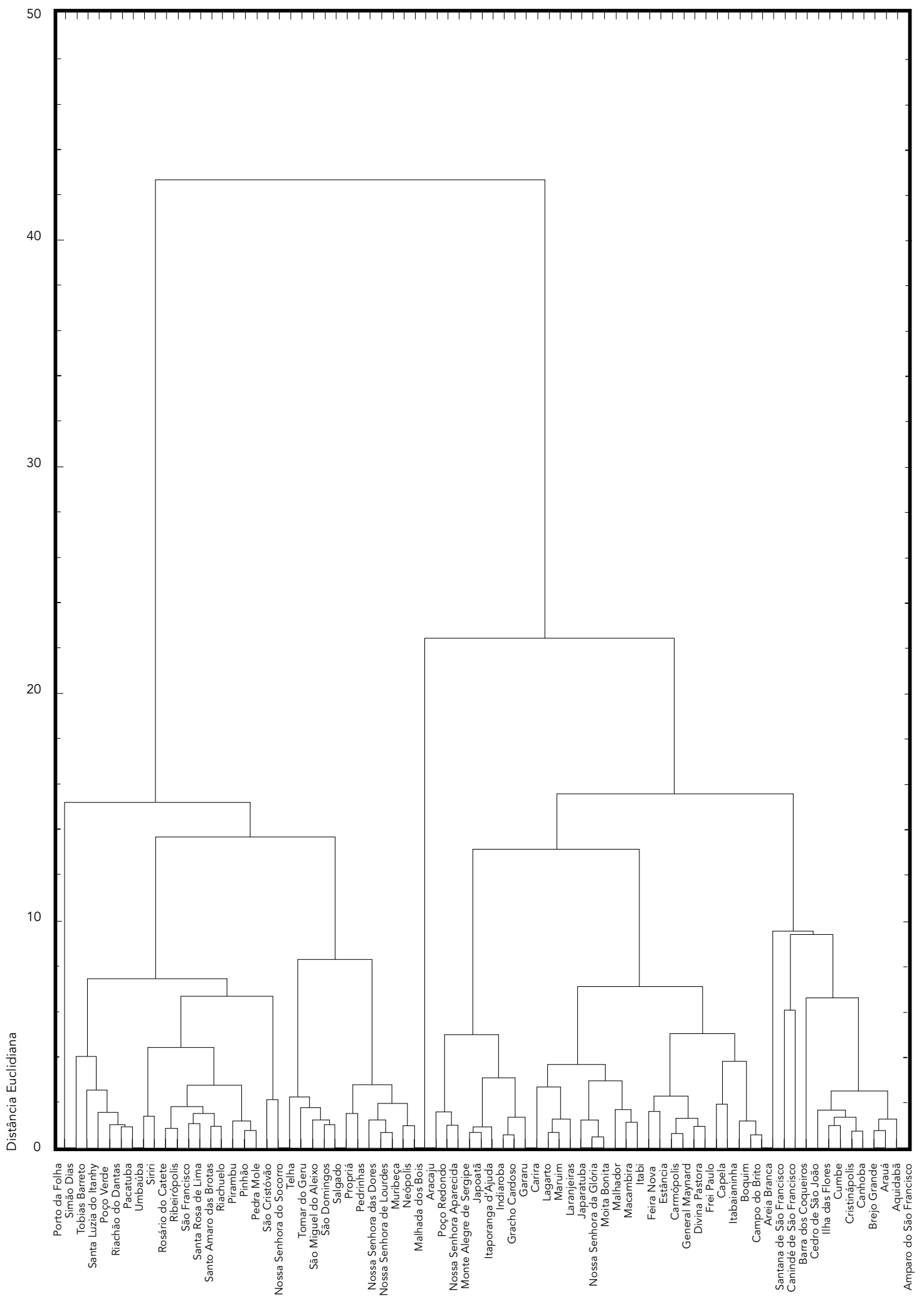


tais dessa região, sendo superada apenas por Fortaleza com apenas $6,51 \%$ dos nascimentos nessa situação. As demais apresentam situação bem diferenciada a exemplo de Maceió, com $12,00 \%$; Salvador, com 9,93\%; Recife, $8,60 \%$; Teresina, 8,29\%; São Luís, 7,95\%; Natal, 7,85\%; e João Pessoa, com 7,81\%.

Os índices apresentados por Sergipe e sua capital, embora ainda não sejam os desejáveis, revelam uma elevação dos padrões de vida em decorrência de políticas públicas adotadas nos últimos dez anos, a exemplo de programas de saúde, de educação e de erradicação da pobreza.

Os índices de baixo peso ao nascer, em Sergipe, como em todas as regiões do país, tendem a ser mais elevados nas áreas geográficas de grande concentração populacional e nos estratos sociais de menor renda 14 . Segundo dados da Pesquisa Estadual de Saúde e Nutrição, a proporção de crianças nascidas com peso inferior a $2.500 \mathrm{~g}$, em 1990, foi de $11,00 \%$, sendo maior na capital, Aracaju (13,00\%), do que no interior do Estado (10,00\%). Já naquela época, havia indícios de que os índices de baixo peso ao nascer nas áreas urbanas eram mais elevados. Para o período estudado, Aracaju teve, em média, um percentual de baixo peso ao nascer de $7,39 \%$, enquanto em Sergipe foi de $6,97 \%$ (Figura 2).

As análises por DRS (Tabela 2 e Figura 2) e por grupamentos de municípios (Tabela 3) permitem evidenciar como o baixo peso ao nascer e as demais variáveis se correlacionam e se distribuem em Sergipe.

Dos sete grupos resultantes da análise de grupamento, dois são resíduos formados pelos municípios de Aracaju e Porto da Folha. Como capital, Aracaju concentra parte expressiva da população sergipana, sendo que na região metropolitana vivem cerca de $40,00 \%$ dos habitantes do Estado. Além disso, os serviços e funções mais especializadas estão lá instalados. Portanto, a cidade centraliza, também, a maior parte dos equipamentos de saúde e de pessoal qualificado, assim como da renda. Aracaju apresentava os maiores IDH $(0,739)$ e IDI $(0,663)$ do Estado e a maior cobertura vacinal $(100,00 \%)$, além de deter o menor índice de mortalidade infantil $(42,89 \%$ o), enquanto a cobertura de prénatal era de apenas $18,75 \%$. Mesmo assim, o percentual de baixo peso ao nascer foi de $7,39 \%$ em um total de 42.721 nascimentos vivos no período, o que corresponde a $25,90 \%$ dos nascimentos de Sergipe no período analisado. Por outro lado, em situação oposta está Porto da Folha, situado no sertão, com 25.664 habitantes (apenas $1,40 \%$ da população estadual, segundo censo do Instituto Brasileiro de Geogra- fia e Estatística 12). Dentre as variáveis estudadas, aparece com o menor IDI do Estado $(0,349)$, assim como a menor cobertura vacinal $(58,03 \%)$. Além disso, apresenta um dos menores IDH $(0,346)$ bem como baixa cobertura de atendimento a gestantes $(6,98 \%)$ e níveis de mortalidade infantil $(77,50 \%)$ que estão entre os mais elevados do Estado. O percentual de baixo peso ao nascer no município, de 1995 a 1998, foi de $6,28 \%$, situação contraditória com os índices anteriores, causada, provavelmente, pelo pequeno número de nascimentos, apenas 2.210 no período estudado (Figura 2).

O Grupo 1 é formado por 19 municípios de diferentes portes populacionais, distribuídos por todo o Estado, formando manchas, sendo uma delas situada no sudoeste, indo desde Tobias Barreto até Pinhão, e outra, na faixa litorânea no nordeste do Estado, desde São Cristóvão até Pacatuba. O Grupo 2 é constituído por 12 municípios, dispersos na faixa central, indo desde Tomar do Geru até Propriá. O Grupo 3 é formado por 9 municípios, sendo a maioria localizada no sertão, a exemplo de Carira, Gararu e Poço Redondo. Na faixa litorânea estão Indiaroba e Itaporanga d'Ajuda. O Grupo 4 apresenta o maior número de municípios (22) dispersos pelas diversas áreas do Estado, mas, com predomínio da área central. É interessante destacar a presença de cidades que dispõem de equipamentos com caráter regional, a exemplo de Lagarto, Estância e Itabaiana. Por fim, temse o Grupo 5 com 11 pequenos municípios espalhados pelo norte, como Canhoba, Aquidabã e Cedro de São João; no leste, como Barra dos Coqueiros; ao sul, Cristinápolis; e no extremo noroeste, Canindé de São Francisco.

Por meio da análise de regressão múltipla foi possível verificar a adequação de cada município ao seu respectivo grupo, feito por um processo probabilístico de reclassificação dos municípios com o uso de funções discriminatórias e lineares, geradas no processo. Na variável baixo peso ao nascer, a análise indica que todos os grupos, estatisticamente, são iguais. Muito embora haja heterogeneidade entre alguns municípios de DRS ou entre municípios de um mesmo grupo resultante do dendrograma, há homogeneidade estatística entre todos os grupos. O mesmo ocorre com as variáveis percentual de vacinação infantil e de moradores com água.

Nas Tabelas 1 e 2 é possível analisar as variáveis nos grupos de municípios inferidos na análise de agrupamentos e nos DRS pelo método de comparações múltiplas. Na análise dos DRS, quanto à variável IDH, observa-se a formação de dois grupos, sendo um deles forma- 
do pelos DRS 1, 2 e 7 e o outro constituído pelos DRS 3, 4, 5 e 6 . A variável mortalidade infantil também vai apresentar dois grupos com situação inversa ao IDH, significando melhor condição. Quanto aos índices de cobertura de pré-natal, constata-se a formação de dois grupos: o primeiro formado pelos DRS 1 e 7, com maior cobertura, e o outro pelos demais $2,3,4$, 5 e 6 em condição inferior.

Nos resultados apresentados para as mesmas variáveis para os grupos resultantes da análise de agrupamento, verifica-se que há uma igualdade aproximada de condições para as variáveis baixo peso ao nascer, mortalidade infantil, vacinação infantil e percentual de moradores com abastecimento de água. Nas variáveis IDH e IDI, os grupos 1, 2 e 4 apresentam condições superiores aos grupos 3 e 5 . Em relação à taxa de alfabetização, observou-se uma semelhança entre os grupos, com exceção para o Grupo 3 que apresentou condição inferior.

Atualmente, segundo informações do SINASC/DATASUS 14, Sergipe está inserido entre os dez menores índices de baixo peso ao nascer dentre as unidades federadas: Minas Gerais (9,27\%), Rio de Janeiro (8,88\%), São Paulo $(8,56 \%)$, Rio Grande do Sul $(8,48 \%)$, Amapá $(8,24 \%)$ Paraná $(7,62 \%)$, Bahia $(7,48 \%)$, Espírito Santo $(7,42 \%)$ Paraíba $(7,42 \%)$. As disparidades interregionais são bem evidentes e reproduzem a situação encontrada entre os diversos países 15 . As regiões periféricas das grandes cidades são depositórias de excluídos, que sofrem de forças deteriorantes. As grandes cidades dos países subdesenvolvidos são cidades críticas. Os pobres não parecem ter sido objeto de preocupação dos economistas espaciais 16,17.

Dos 75 municípios analisados, 11 apresentaram índices de baixo peso ao nascer superior a $8,00 \%$, dentre os quais os mais elevados foram Campo do Brito (10,10\%), Laranjeiras $(9,08 \%)$ e Nossa Senhora Aparecida $(8,75 \%)$. Esse último apresenta um dos menores IDH do estado, enquanto Laranjeiras, situado na Zona do Cotinguiba, tem altos níveis de pobreza, tendo em vista a concentração da terra e a má distribuição de renda. Por outro lado, nove municípios apresentaram índices inferiores a 5,80\%. Esses municípios apresentam IDH maior do que 0,400 , sendo que dentre eles, Itabaianinha tem melhor situação $(0,507)$ (Figura 2$)$.

Essa situação evidencia a aproximação com índices característicos do mundo subdesenvolvido. Sete municípios apresentaram índices inferiores a 5,00\%: Santa Rosa de Lima (5,00\%), Telha $(4,30 \%)$, Itabaianinha $(4,00 \%)$, Pirambu $(3,98 \%)$, Cumbe $(3,90 \%)$, Cedro de São João $(3,85 \%)$ e São Francisco $(3,42 \%)$ o que os apro-
Figura 2

Distribuição percentual do baixo peso ao nascer. Sergipe, Brasil, 1995/1998.
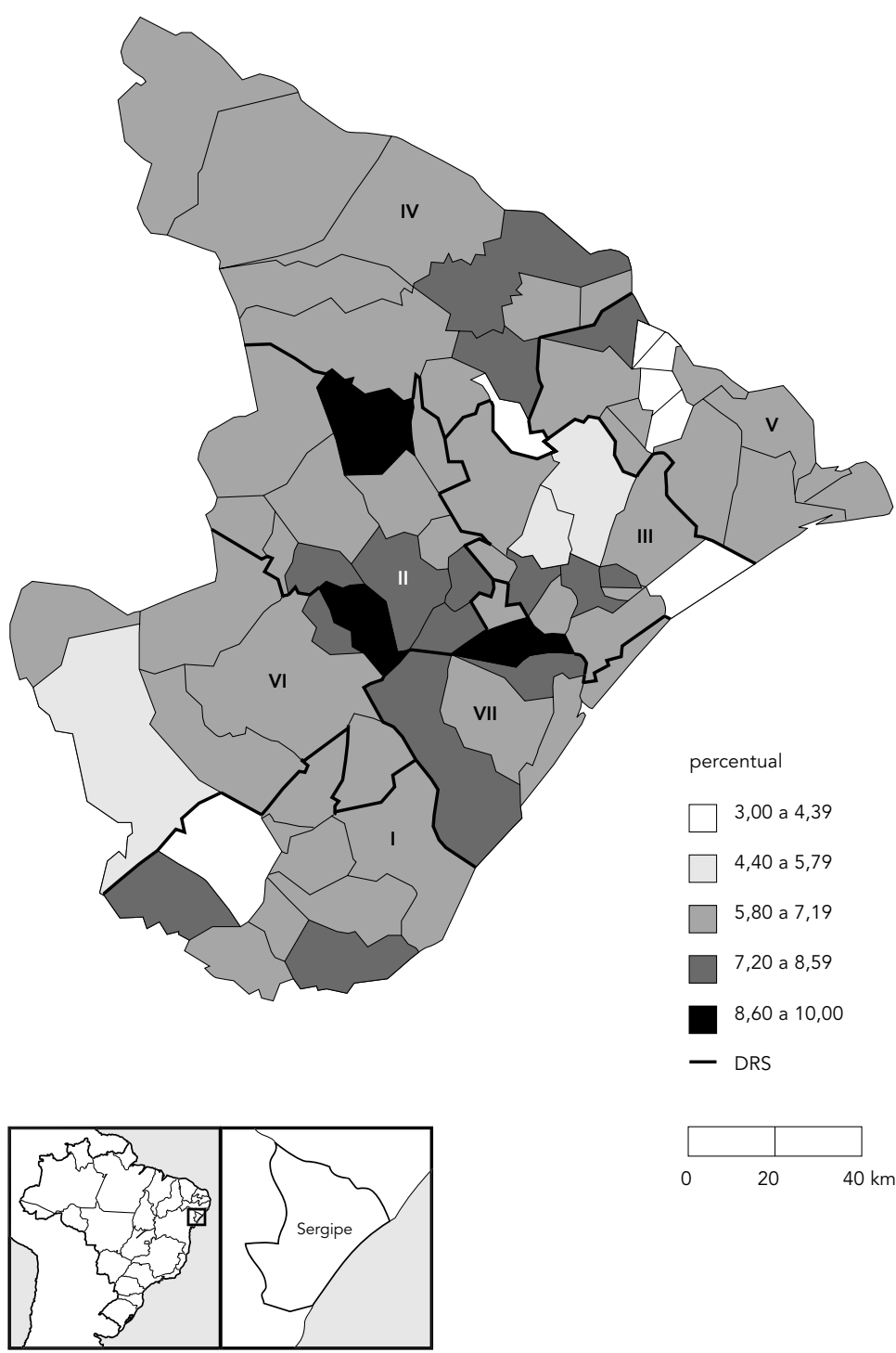

Fonte: SINASC/DATASUS.

ximam da situação apresentada por lugares mais desenvolvidos, fato que não corresponde à realidade. De fato, há que se questionar tal situação em função do pequeno número de nascimentos.

Maia 18, estudando baixo peso ao nascer em Minas Gerais, encontrou taxa de $10,00 \%$, dados superiores aos encontrados por Costa \& Gotlieb 19 em municípios paulistas: Marília (7,40\%), São José dos Campos (7,80\%) Santo André (6,80\%) 
e Pariquera-Açu (6,60\%). Itararé apresentou a maior prevalência $(10,40 \%)$. Em Salvador, as áreas periféricas e centrais deterioradas mostravam indicadores de pior qualidade 20 .

Estudos desenvolvidos por Sadeck et. 21, na maternidade do Hospital das Clínicas de São Paulo, Universidade de São Paulo, sobre a variação secular do peso de recém-nascidos, consideram o peso ao nascer um dos principais indicadores de saúde de uma população, pois é um forte previsor da morbidade e da mortalidade neonatais. Afirmam ainda que melhoras econômicas no país são responsáveis pelo aumento secular do peso dos recém-nascidos, confirmando a influência da situação sócio-econômica no baixo peso ao nascer.

Atualmente, as questões postas pela globalização apontam também para as possibilidades de fortalecimento da miséria e da exclusão. Não deve ser por acaso que a pobreza e as desigualdades voltam à ordem do dia, tanto nos círculos acadêmicos de países centrais, como nos discursos dos próprios organismos internacionais 22 , causando preocupações que resultam na adoção de políticas públicas compensatórias 7 .

\section{Resumo}

O estudo analisa a distribuição espacial do baixo peso ao nascer em Sergipe, Brasil, avaliando o evento como indicador de saúde. A análise foi feita a partir de 166.312 nascimentos vivos dos 75 municípios, numa abordagem ecológica. Foram utilizados quatro métodos: clusters analysis, análise de correlação, análise de regressão múltipla e multiple comparisons (Tukey). $O$ estudo evidencia homogeneidade interna dos municípios nos distritos de saúde e nos grupos resultantes do dendrograma. Considerando 10,00\% de perda de informação, identifica-se a presença de sete grupos, sendo dois resíduos (Aracaju e Porto da Folha). A evolução do baixo peso ao nascer, em Sergipe, é declinante (média de 6,97\% no período) e reflete tímida melhoria da qualidade de vida. As suas causas indicam relações com o ambiente e são influenciadas pelos padrões de ocupação do espaço. O percentual do baixo peso ao nascer é útil como indicador de risco individual, porém, como indicador coletivo de saúde, não parece ser capaz de expressar diferenças entre regiões que não apresentam variabilidade acentuada entre si.

Distribuição Espacial; Baixo Peso ao Nascer; Eqüidade
Este estudo evidenciou que a evolução das taxas do baixo peso ao nascer, em Sergipe, reflete a melhoria nos indicadores de saúde, no período. A queda nos índices do baixo peso ao nascer pode estar relacionada à melhoria da qualidade de vida, porém, não foram evidenciadas grandes diferenças entre os municípios, os grupos de DRS e nem entre grupos resultantes do dendrograma.

Os índices de baixo peso ao nascer, ao se apresentarem elevados nos municípios mais populosos e nas grandes metrópoles, onde a maior concentração de pobreza reflete a complexidade e a diferenciação das condições de vida presentes nesses centros, podem funcionar como bons indicadores coletivos de saúde e de qualidade de vida. Talvez, estudos dessa natureza ganhem maior expressão se a escala de análise utilizada for aquela que permita observar diferenciações internas, como os setores censitários ou o bairro de residência, evidenciando as diversas nuances sócio-econômicas presentes no espaço. Assim, novos estudos se fazem necessários a fim de avaliar a utilização dos índices de baixo peso ao nascer como indicador coletivo de saúde.

\section{Colaboradores}

R. Q. Gurgel realizou as revisões do artigo e participou em todas as fases do estudo. I. M. O. Dias trabalhou em todas as fases do estudo. V. L. A. França participou das discussões em todas a fases do projeto e elaborou os mapas e figuras. D. F. N. Castañeda fez a análise estatística do estudo. 


\section{Referências}

1. Eisner V, Brazie JV, Pratt MW, Hexter AC. The risk of low birth weight. Am J Public Health 1979; 69:887-93.

2. Beal VA. Assessment of nutritional status in pregnancy-11. Am J Clin Nutr 1981; 34:691-6.

3. Belfort P. Crescimento intra-uterino retardado. In: Belfort P. Medicina perinatal. São Paulo: Editora Manole; 1983. p. 211-5.

4. McCormick M. The contribution of low birth weight to infant mortality and childhood morbidity. N Engl J Med 1985; 312:82-90.

5. Kramer MS. Determinants of low weight: methodological assessment and meta-analysis. Bull World Health Organ 1987; 65:663-737.

6. World Health Organization. The incidence of low birth weight: a critical review of available information. World Health Stat Q 1980; 33:197-244.

7. Organización Panamericana de la Salud. La salud en las Américas. v. I. Washington DC: Organización Panamericana de la Salud; 1998. (Publicación Científica n. 569).

8. Boldman R, Reed DM. World wide variation in low birth weights. In: Reed DM, Stanley F, editors. Epidemiology of prematurity. Baltimore: Urban and Swartzenberg; 1997. p. 39-51.

9. Victora CG, Grassi PR, Schmidt AM. Situação de saúde da criança em área da região sul do Brasil, 1980-1982: tendências temporais e distribuição espacial. Rev Saúde Pública 1994; 28:423-32.

10. Puffer RR, Serrano CV. Patterns of birth weight. Washington DC: Pan American Health Organization; 1987. (Scientific Publication n. 504).

11. Secretaria de Estado da Saúde. Modelo assistencial de Sergipe. Sistema Unificado e Descentralizado de Saúde (SUDS)/Sergipe. Aracaju: Secretaria de Estado da Saúde; 1987.

12. Instituto Brasileiro de Geografia e Estatística. Censo demográfico, 2000. Rio de Janeiro: Instituto Brasileiro de Geografia e Estatística; 2002.

13. Bisquerra A. Introducción conceptual al analisis multivariable. Barcelona: Ediciones PPU; 1989.
14. Ministério da Saúde. Sistema de Informações sobre Nascidos Vivos - SINASC. http://www.datasus.gov.br (acessado em 22/Jan/2002).

15. Curi PR. Agrupamento de países segundo padrão de vida. Rev Saúde Pública 1993; 27:127-34.

16. Santos M. Por uma economia política da cidade. São Paulo: Editora Hucitec; 1994.

17. Santos M. O mercado não resolve tudo. Cadernos do Terceiro Mundo 1997; 200:20-3.

18. Maia MAC. Caracterização dos nascidos vivos hospitalares no primeiro ano de implantação do subsistema de informação sobre nascidos vivos em município de Minas Gerais. Inf Epidemiol SUS 1997; 31:6-14.

19. Costa CE, Gotlieb SLD. Estudo epidemiológico do peso ao nascer a partir da Declaração de Nascido Vivo. Rev Saúde Pública 1998; 32:328-34.

20. Paim JS. Abordagens teórico-conceituais em estudos de condições de vida e saúde: notas para reflexão e ação. In: Barata RB, organizador. Condições de vida e situação de saúde. Rio de Janeiro: ABRASCO; 1997. p. 7-30.

21. Sadeck LSR, Barros JCR, Ramos JLA. Variação secular do peso de recém-nascidos do Hospital das Clínicas de São Paulo em quatro períodos nos últimos 50 anos. Pediatria (São Paulo) 2000; 22:1420.

22. World Bank. World development report 1990. Washington DC: World Bank; 1991.

23. Fundo das Nações Unidas para a Infância. Relatório da situação da infância brasileira. Brasília: Fundo das Nações Unidas para a Infância; 2001.

24. Empresa de Desenvolvimento Sustentável do Estado de Sergipe, Secretaria de Planejamento e Tecnologia de Sergipe. Projeto São José, Aracaju: Seplantec-Pronese. Aracaju: Empresa de Desenvolvimento Sustentável do Estado de Sergipe, Secretaria de Planejamento e Tecnologia de Sergipe; 2001.

Recebido em 18/Nov/2003

Versão final reapresentada em 15/Fev/2005

Aprovado em 01/Mar/2005 